University of Wollongong

Research Online

Australian Institute for Innovative Materials -

Papers

Australian Institute for Innovative Materials

$1-1-2014$

\title{
Graphene oxide dispersions: tuning rheology to enable fabrication
}

Sina Naficy

University of Wollongong, snaficy@uow.edu.au

Rouhollah Jalili

University of Wollongong, rjalili@uow.edu.au

Seyed Hamed Aboutalebi

University of Wollongong, sha942@uowmail.edu.au

Robert A. Gorkin III

University of Wollongong, rgorkin@uow.edu.au

Konstantin Konstantinov

University of Wollongong, konstan@uow.edu.au

See next page for additional authors

Follow this and additional works at: https://ro.uow.edu.au/aiimpapers

Part of the Engineering Commons, and the Physical Sciences and Mathematics Commons

Research Online is the open access institutional repository for the University of Wollongong. For further information contact the UOW Library: research-pubs@uow.edu.au 


\title{
Graphene oxide dispersions: tuning rheology to enable fabrication
}

\author{
Abstract \\ Here, we show that graphene oxide (GO) dispersions exhibit unique viscoelastic properties, making them \\ a new class of soft materials. The fundamental insights accrued here provide the basis for the \\ development of fabrication protocols for these two-dimensional soft materials, in a diverse array of \\ processing techniques. \\ Keywords \\ fabrication, enable, graphene, rheology, oxide, tuning, dispersions \\ Disciplines \\ Engineering | Physical Sciences and Mathematics \\ Publication Details \\ Naficy, S., Jalili, R., Aboutalebi, S. Hamed., Gorkin III, R. A., Konstantinov, K., Innis, P. C., Spinks, G. M., \\ Poulin, P. \& Wallace, G. G. (2014). Graphene oxide dispersions: tuning rheology to enable fabrication. \\ Materials Horizons, 1 (3), 326-331.

\section{Authors} \\ Sina Naficy, Rouhollah Jalili, Seyed Hamed Aboutalebi, Robert A. Gorkin III, Konstantin Konstantinov, Peter \\ C. Innis, Geoffrey M. Spinks, Philippe Poulin, and Gordon G. Wallace
}




\section{Graphene Oxide Dispersions: Tuning Rheology to Enable Fabrication}

Sina Naficy ${ }^{\ddagger} a$, Rouhollah Jalili ${ }^{\ddagger} a$, Seyed Hamed Aboutalebi ${ }^{\ddagger}$, Robert A. Gorkin III ${ }^{a}$, Konstantin Konstantinov ${ }^{b}$, Peter C Innis ${ }^{a}$, Geoffrey M. Spinks ${ }^{a}$, Philippe Poulin $^{c}$ and Gordon G. Wallace ${ }^{a} *$

Here, we show that graphene oxide (GO) dispersions exhibit unique viscoelastic properties which constitute them as a new class of soft materials. The fundamental insights accrued here provide the basis for the development of fabrication protocols for these two dimensional soft materials, in a diverse array of processing techniques.

The recent discovery of liquid crystalline behaviour in graphene oxide (GO) dispersions in water, ${ }^{1-5}$ and various organic solvents, ${ }^{6,7}$ can be implemental in guiding material assembly at atomic level through $\pi-\pi$ stacking and hydrogen bonding interactions..$^{1,5,6,8}$ Original rheological behaviour is expected, since monolayer GO dispersions constitute a new class of soft material with rich unique properties in between rigid 2D nanoplatelets and $1 \mathrm{D}$ polymers. ${ }^{9}$ Probing and quantifying this dynamic behaviour will be instrumental in generating novel applications, such as coatings, fillers and molecular electronics by advancing our fundamental knowledge of soft materials. ${ }^{10}$ Despite the importance for fundamental, practical, and industrial applications, the rheological behaviour of GO monolayer dispersions and its origin remains an unexplored area. This, in turn, hinders the development of fabrication protocols for two dimensional soft materials, including GO dispersions. Moreover, fabricating practical devices in large-scale with advanced architectural design is still a big challenge, unresolved.

Likewise, formulation of functional composite inks for industrially scalable fabrication methods such as electrospraying, spray coating, and printing techniques, is always hindered by the high concentration of supporting media (such as graphene, GO, LCs and polymers). Therefore, low concentration of the supporting media is desirable to simultaneously permit processing while making the preparation of composite inks with high concentrations of multi-functional materials possible. This challenge, if resolved, can be used to create geometrically complex multi-functional $3 \mathrm{D}$ architectures fabricated for use in areas such as printed electronics, organic field effective transistors and 3 D bionic scaffolds.

Here, with the aim of investigating the rheological behaviour of $\mathrm{GO}$ to enable further processing, fabrication and integration of $\mathrm{GO}$ into complex architectures, we employed ultra large $\mathrm{GO}$ sheets as a model material with high aspect ratio $\left(\sim 45000, \mathrm{Fig} . \mathrm{S}_{1}\right)$ to study the phase transitions from the so-called isotropic phase to liquid crystal (LC) phase and finally to a LC gel phase at higher loadings. We demonstrate that GO dispersions exhibit unique viscoelastic behaviour, wherein the rheological behaviour varies considerably with dispersion concentration. We show that finite yield stress in ultra large GO dispersions occurs at a critical concentration approximately three orders of magnitude lower than the theoretical value for colloidal suspensions $\left(\phi_{\text {this study }} \approx 2.2 \times 10^{-4}\right.$ vs. $\left.\phi_{c} \approx 0.5\right)$ enabling the processing of this $2 \mathrm{D}$ material at the lowest concentration ever reported for any dispersions allowing the self-assembly of multifunctional architectures. Our experiments reveal that there are four distinct regions: viscoelastic liquid, a transition state consisting of viscoelastic liquid and viscoelastic soft solid, viscoelastic soft solid (behaving like a solid below yield stress yet flow readily above a yield stress) ${ }^{11}$ and viscoelastic gel. Each of these unbinding regions were found amenable to unique processing techniques, some of which never before shown possible in case of $\mathrm{GO}$, warranting the process of this fascinating material based on its inherent complex flow properties.

Control over the rheological properties can be achieved by adjusting the volume fraction (concentration) of GO particles. To this end, we probed, as plotted in Fig. 1 and S2, the flow behaviour of a series of GO dispersions by the cone-plate method (see the provided Supporting Information for detailed information on the experimental section). The elastic $G^{\prime}$ (storage) and viscous $G^{\prime \prime}$ (loss) moduli of GO dispersions were determined as a function of frequency at a constant strain amplitude of 0.01 . 

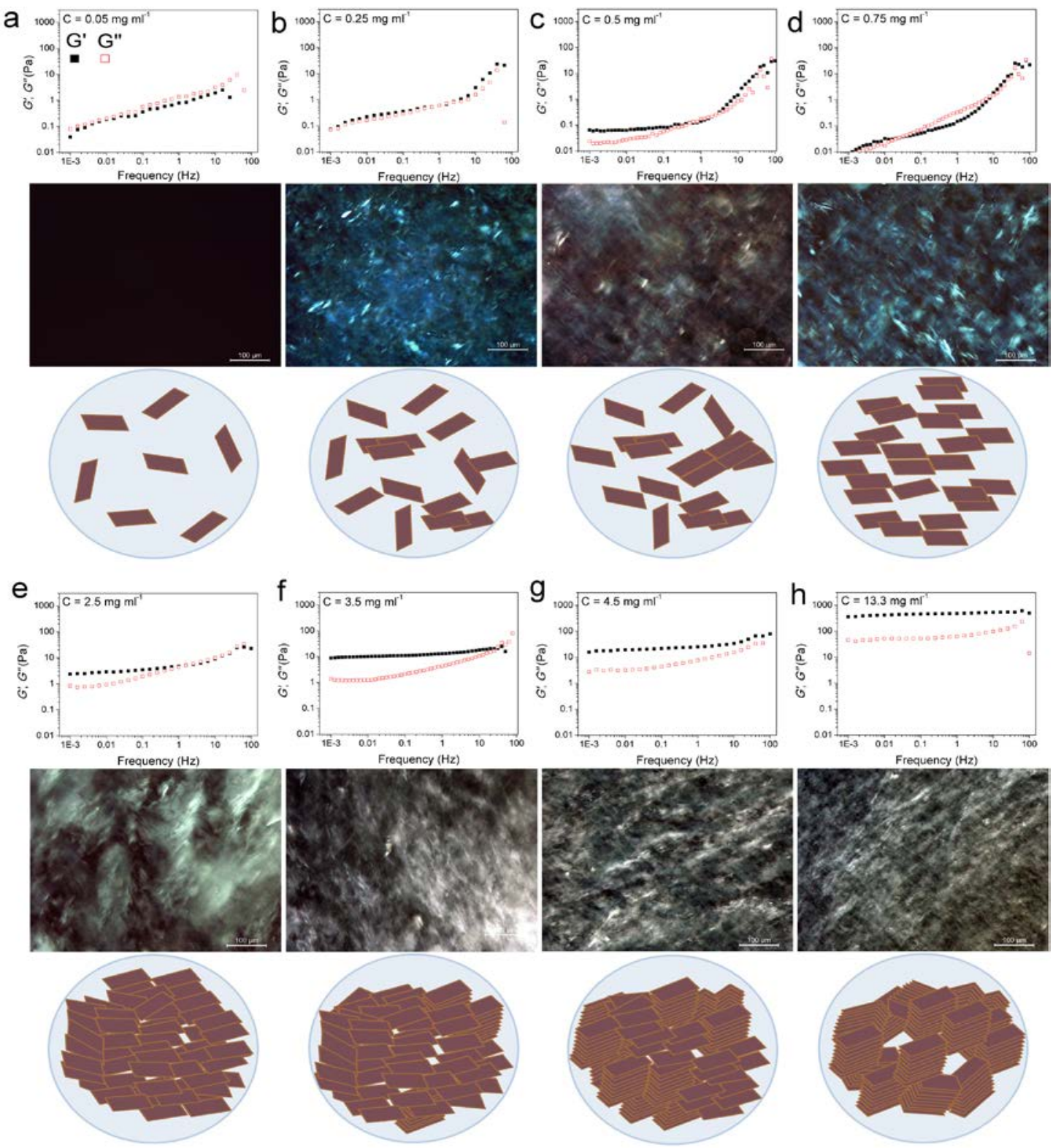

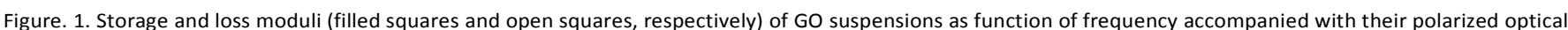

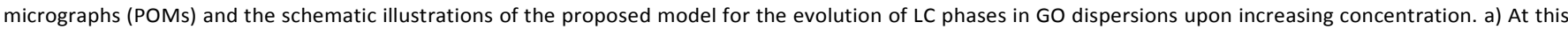

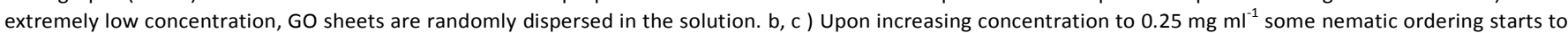

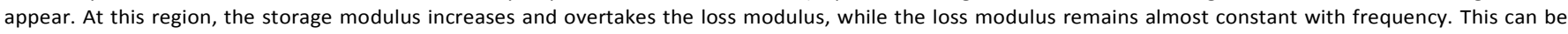

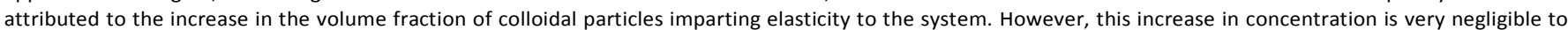

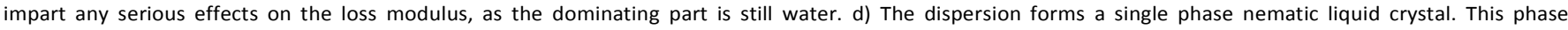

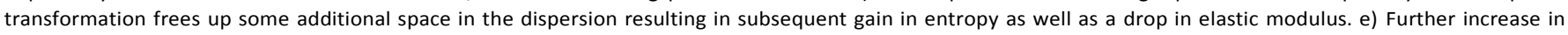

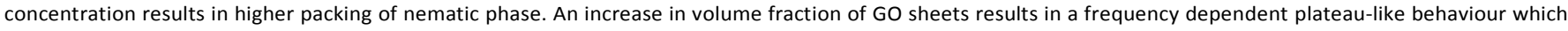

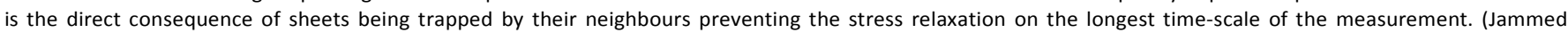

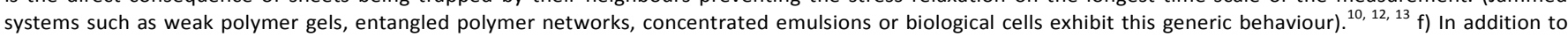

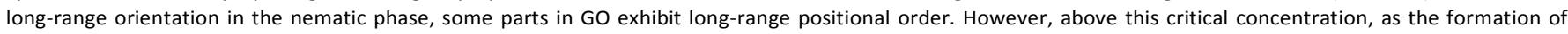

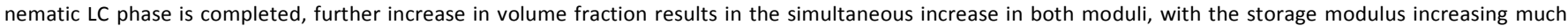
faster than the loss modulus. $g$ and $h$ ) smaller monodomains are formed associated to exceptional increase in elastic modulus. 
Quite interestingly, even at very low concentrations (as low as 0.05 and up to $0.25 \mathrm{mg} \mathrm{ml}^{-1}$ ), previously thought to be a completely isotropic and viscous phase, ${ }^{1,5}$ a considerable elastic component still exists. Over all time-scales, in the low concentration region $(<0.25 \mathrm{mg}$ $\mathrm{ml}^{-1}$ ), the dispersions exhibited a liquid-like response with $G^{\prime \prime}$ being slightly higher than $G^{\prime}$ suggesting a viscoelastic liquid like behaviour similar to colloidal suspensions near their crystallization point at volume fractions $\phi_{c} \sim 0.5 .{ }^{10}$ The dominance of $G^{\prime \prime}$ along with a large $G^{\prime}$ suggests the existence of so-called crowding or jamming. ${ }^{10}$ This implies that the configurational rearrangement necessary for equilibration cannot be achieved within the time framework studied here. In our system, jamming resulted in the development of a finite yield stress ${ }^{12}$ at concentrations approximately three orders of magnitude lower than the theoretical value for spherical colloidal suspensions $\left(\phi_{\text {this study }} \approx 2.2 \times 10^{-4} \mathrm{vs} . \phi_{c} \approx 0.5\right){ }^{10}$ Jamming at such low concentrations is due to the extremely large aspect ratio of ultra large wrinkled GO sheets used in this study (Fig. S1). The dominance of the viscous part in all time scales (liquid-like behaviour) while having a considerable elastic and viscosity components, in such low concentrations, has a direct impact on processability and fabrication. Specifically this behaviour enables us to easily electrospray and spraycoat with a high level of control (Table $\mathrm{S}_{1}$ and Fig. 2), while eliminating the drift perturbations typically associated with spraying of viscous fluids with no elastic component. These rheological features also allow us to spray at extremely low concentrations leading to low mass loading levels deposited at the substrate surface and subsequently enabling the fabrication of ultra-transparent GO thin films (Transparency of $98.04 \%$ and $95.4 \%$ at $633 \mathrm{~nm}$ for 1 and two-time coated glass slides after partial reduction by heat-treatment at $220^{\circ} \mathrm{C}$ corresponding to approximately 1 layer of GO per run (Fig. 2d)). It should be noted that such a level of control is usually achieved through employing highly time consuming Langmuir-Blodgett method. ${ }^{14,}{ }^{15}$ Spray coating of GO dispersions has great potential in many industrial processes such as electronics, painting, microencapsulation, electroemulsification, fine powder production, or micro- and nano-thin film deposition. ${ }^{16}$

In the second region ( $\mathrm{GO}$ concentration $\sim 0.25$ up to $0.75 \mathrm{mg} \mathrm{ml}^{-1}$ ), GO dispersions show biphasic behaviour (coexistence of both isotropic and nematic phase). Here a viscoelastic behaviour which is in contrast with normal lyotropic liquid crystal phases can be observed. ${ }^{17}$ In typical lyotropic liquid crystals, the viscous part $\left(G^{\prime \prime}\right)$ is often dominant at large time-scales or towards the lower frequencies. ${ }^{17}$ On the other hand, here $G$ ' appears to be higher than $G$ " for GO dispersions falling in this region at large time-scales. This behaviour suggests the crowding of particles and consequently gelation of the system as a result of repulsive interactions experienced by neighbouring GO sheets which is also consistent with the yield point observed at this region (Fig. 3a). Therefore, over long-time scales, GO dispersions respond more like a viscoelastic soft solid as the "long-range" rearrangements (convolutions) are very slow. However, above the $G^{\prime}$ $G^{\prime \prime}$ crossover point, in an intermediate time scale, $G^{\prime}$ was found to be lower than $G$ " suggesting a dominant viscous behaviour (liquid-like) implying that "short-range" rearrangements rapidly occur. Nevertheless, up to the concentration of $0.75 \mathrm{mg} \mathrm{ml}^{-1}$, the difference is not considerable and the zones are not yet well-defined. These fluid properties are ideal for inkjet printing as the ink formulation should be designed to quickly regain viscosity and hence shapes (rearrange the mesogens) once they are printed on the surface to preserve the printed structure for accurate reproduction. ${ }^{18}$ Importantly, this new finding enables us to formulate stable inks from pure GO dispersions without the need of any binders or additives to impart the dominant elastic components required for ink-jet printing (Fig. 2 e). It should also be noted that although the size of our GO sheets (average diameter $37 \mu \mathrm{m}$ ) are much larger than the safe zone ${ }^{19}$ criterion for our inkjet printer nozzles ( $1 \mu \mathrm{m}$ for a $50 \mu \mathrm{m}$ nozzle), we were able to successfully print GO without any clogging side effects as a consequence of the highly flexible nature of GO sheets (Fig. S1). The inkjet printing of binder free GO will enable the fabrication of high quality electrode materials that are critical for use in electronic applications such as organic field effective transistors (OFETs).

At higher concentrations (above 0.75 up to $2.5 \mathrm{mg} \mathrm{ml}^{-1}$ ), the storage and loss moduli are completely and clearly well-separated from each other (Fig. 1d and 1e). This is the concentration range at which GO dispersions form single phase nematic liquid crystals. The two dimensional GO sheets form a tenuous network architecture with a very dominant elastic part in spite of the considerably low concentration. The $G^{\prime}-G^{\prime \prime}$ crossover point begins to shift towards higher frequencies. The rheological behaviour of $\mathrm{GO}$ dispersions at concentrations as high as $2.5 \mathrm{mg} \mathrm{ml}^{-1}$ resembles that of soft glassy materials (SGMs), liquid crystals, and/or weak gels. ${ }^{10,} 20$ This rheological behaviour suggests that the processing of $\mathrm{GO}$ dispersions is possible with established fabrication techniques usually reserved for processing weak gel-like materials (such as the wet-spinning approach). ${ }^{1,21-24}$ However, in contrast to cross-linked biopolymers ${ }^{10}$ and biological gels ${ }^{13}$ no strain stiffening is observed (Fig. 3 b), and unlike gels of other disk-like colloids a fully frequency-dependent elastic modulus is not observed either (Fig. 1e). ${ }^{25}$ Another interesting aspect is the ability of these dispersions to retain their structure at very short time scales. 
a

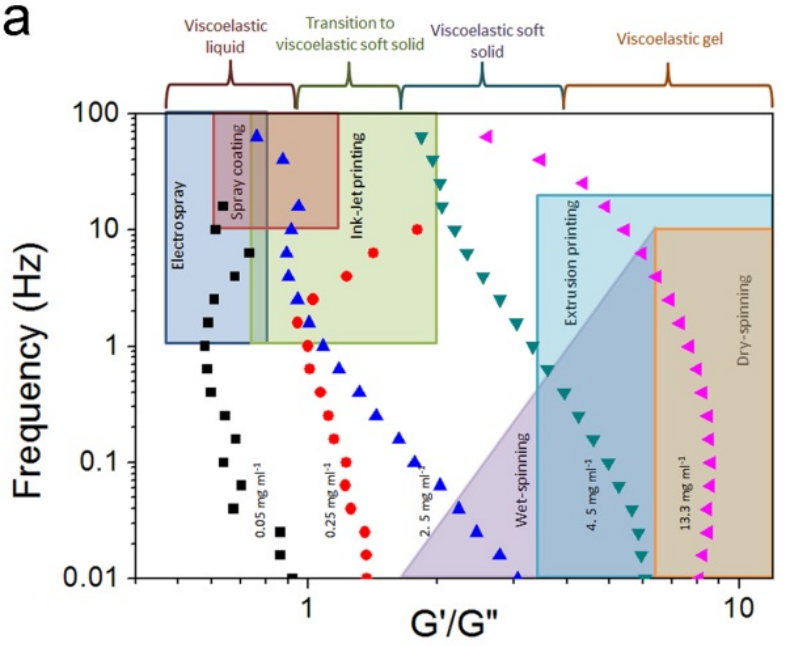

b

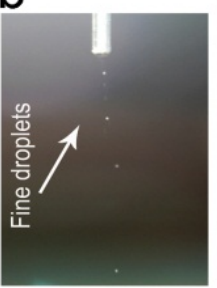

C
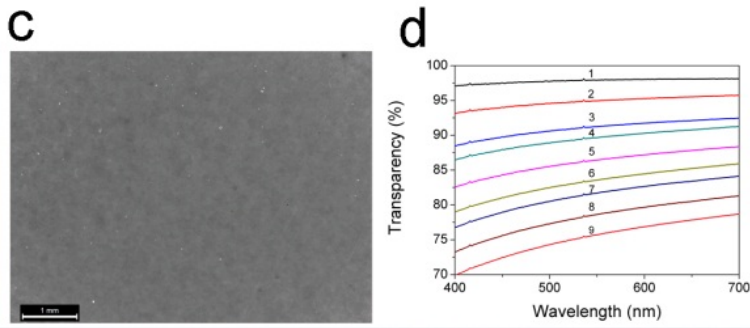

e
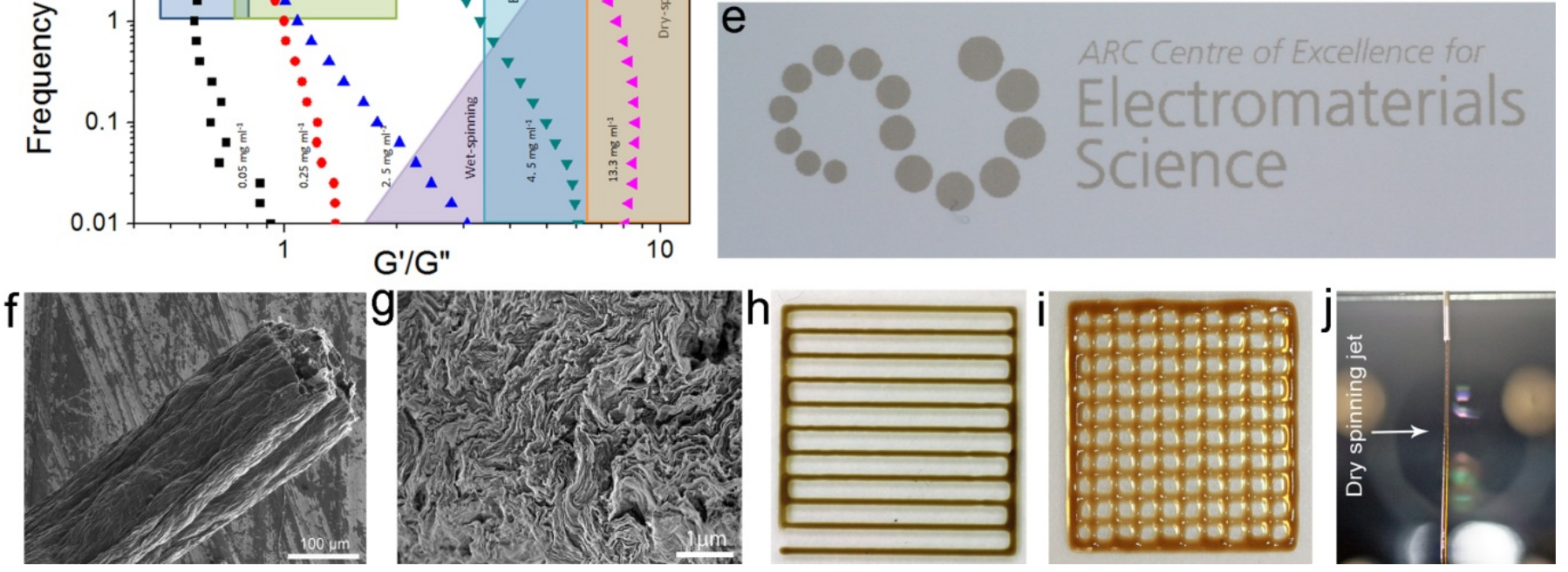

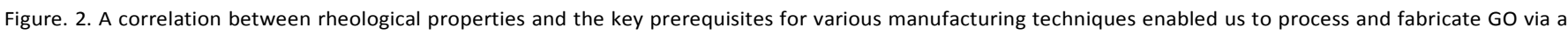

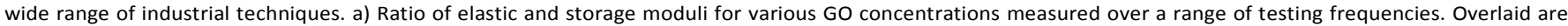

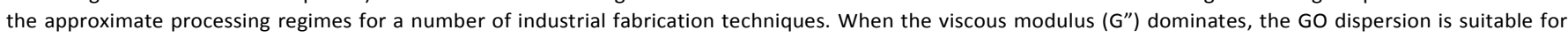

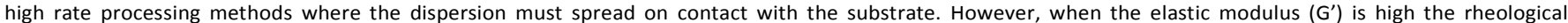

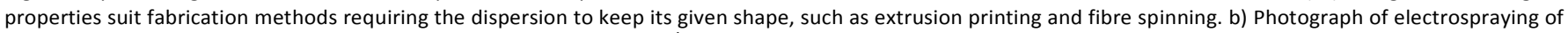

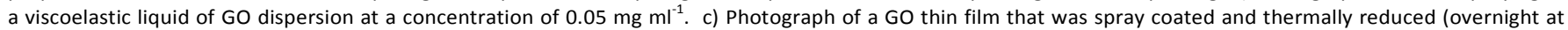

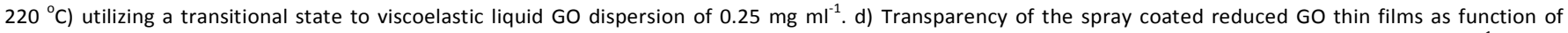

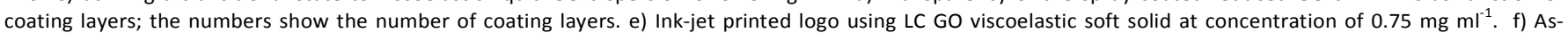

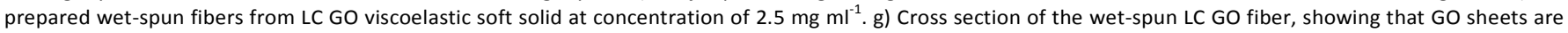

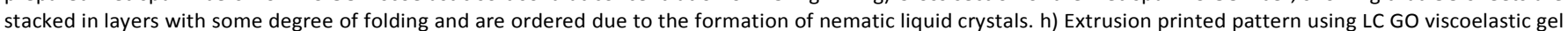

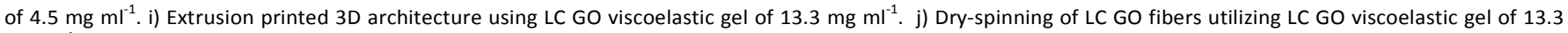
$\mathrm{mg} \mathrm{ml}^{-1}$.

As concentration increases up to $4.5 \mathrm{mg} \mathrm{ml}^{-1} \mathrm{G}^{\prime}$ and $G^{\prime \prime}$ become fully distinct with $G^{\prime}$ reaching $15-77 \mathrm{~Pa}$ at $4.5 \mathrm{mg} \mathrm{m}^{-1}$ (depending on frequency, Fig. 1f). This behaviour is slightly different to polymer networks and resembles the rheological characteristics of cells or SGMs. ${ }^{10,26}$ Purely elastic polymer networks exhibit a completely frequency-independent storage modulus plateau even at low frequencies. ${ }^{10,26}$ Similar to cells however, ${ }^{10} \mathrm{GO}$ dispersions at this region show power-law rheology with a weak non-universal exponent (i.e. the storage modulus increases slowly with frequency). In this region, it is only at very short time scales that the viscous part dominates. The magnitude of storage modulus increases with increasing concentration (volume fraction) and the crossover point $\left(G^{\prime}=G^{\prime \prime}\right)$ shifts to time scales as short as 0.01-0.1 sec as the network becomes more robust and exhibits a gel-like behaviour (Fig. $1 \mathrm{~g}$ ). This behaviour might suggest structural changes even though the rheological properties of liquid crystals of disk like particles are not simply correlated to the phase behaviour of the materials. ${ }^{20,25,27}$ Upon increasing the concentration above 0.75 , the nematic domains (with same orientation and brightness) decrease in size, this in turn, could contribute to substantial increase in the elastic modulus associated to a greater density of defects. ${ }^{28}$

At even higher concentrations (up to $13.35 \mathrm{mg} \mathrm{ml}^{-1}$ ), no $G^{\prime}-G^{\prime \prime}$ crossover was observed in the frequency range studied here, resembling a gel-like (cells or SGMs) behaviour with an extraordinarily high elastic modulus of 350-490 $\mathrm{Pa}$. The measured storage modulus at $13.35 \mathrm{mg} \mathrm{ml}^{-1}$ was considerably higher than the calculated elastic 


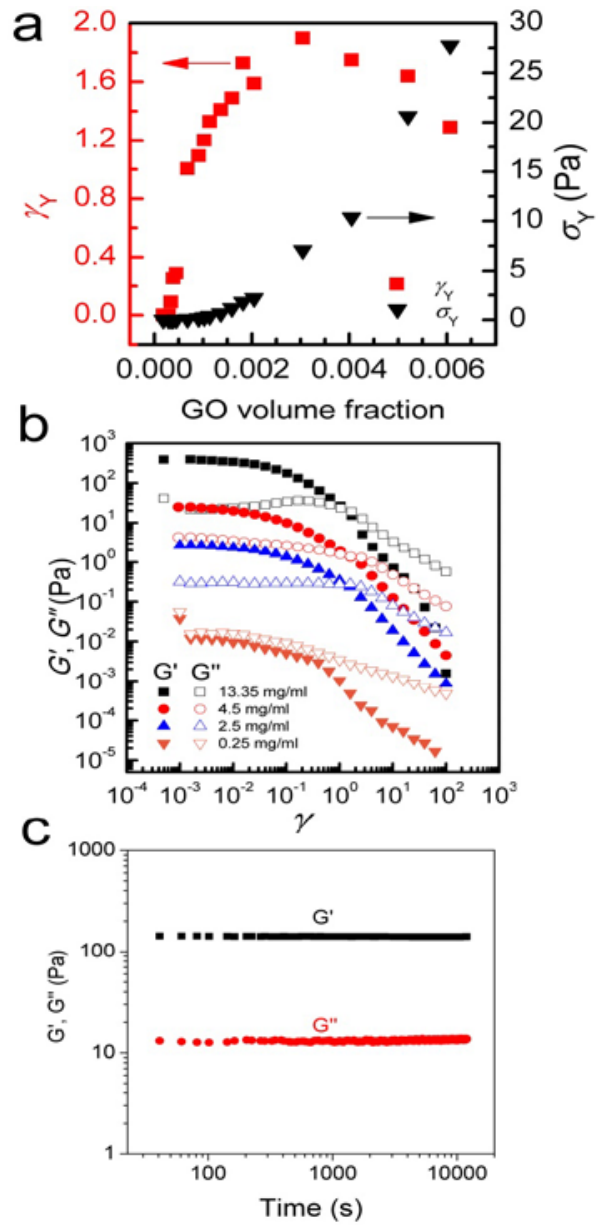

Figure. 3. Interpretation of rheological behaviour of LC GO dispersions. a) Yield strain $\left(\nu_{\mathrm{Y}}\right)$ and yield stress $\left(\sigma_{\mathrm{Y}}\right)$ of various GO suspensions determined at different $\mathrm{GO}$ volume fractions. Yield point is considered as where the storage and loss moduli intercept when measured as a function of frequency. b) Storage (elastic) and loss (viscous) moduli of GO suspensions at the frequency of $0.01 \mathrm{~Hz}$ at different strains. No strain-stiffening can be observed even at very high concentrations which is consistent with SGM rheology generally exhibiting yielding and plasticity. However in contrast to soft glassy rheology (SGR), c) no aging after shear rejuvenation/fluidization can be observed.

modulus of SWNT suspensions at the same concentration ( $\sim 60 \mathrm{~Pa}) .{ }^{29}$ GO dispersions at this concentration range, are therefore, viscoelastic liquid crystals gel that can flow after a yield point. Furthermore, there are some unique characteristics that our as-prepared LC GO viscoelastic gels exhibit such as the anisotropy arising from having a liquid crystal network, and the exceptional uniformity of the network structure. Therefore, GO dispersions prepared in this region enabled us to process GO for the first time in a range of industrial processes such as gel-extrusion printing and dry spinning that are most beneficial for many industrial applications (Fig. 2 h-j). During gelextrusion through a nozzle (i.e. extrusion printer nozzle), the loss modulus will become dominant (Fig. 3b, strain thinning effect), allowing for the dispersion to easily be extruded. However, when the LC GO viscoelastic gels leaves the nozzle (at low strain), the physical sheet entanglement forces them to regain their high elasticity. The high elasticity of LC GO viscoelastic gels retains the printed structure and makes it possible to print fine lines or $3 \mathrm{D}$ architectures (Fig. $2 \mathrm{~h}, \mathrm{i}$ ).
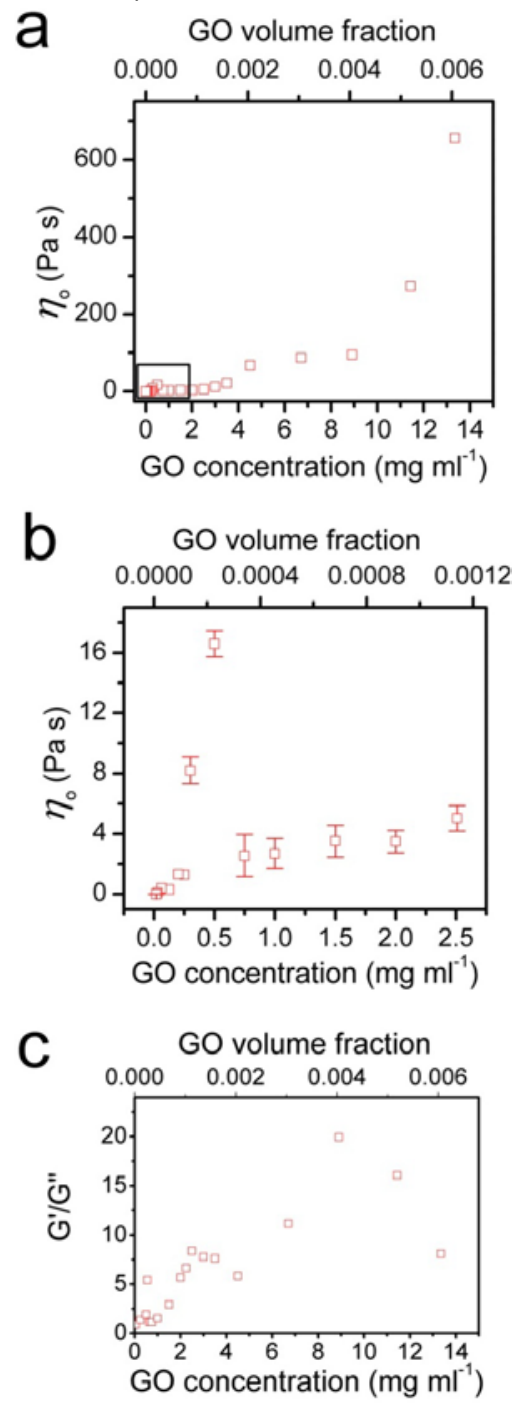

Figure. 4. Viscosity and the ratio of loss and storage moduli of GO dispersions at different GO concentrations. a) Viscosity $\left(\dot{\gamma}=0.01 \mathrm{~s}^{-1}\right)$ as a function of GO volume fraction. Increasing the concentration of $\mathrm{GO}$ results in an overall increase in viscosity until a peak at $\phi \sim 2.3 \times 10^{-4}\left(0.5 \mathrm{mg} \mathrm{ml}^{-1}\right)$. However as depicted in b) a sudden drop in viscosity as a result of ordering happens at higher concentrations. c) The same general trend also happens in the case of the ratio between loss and storage moduli. Moreover, at almost all concentrations, except in the very beginning, the ratio of $G^{\prime}$ to $G^{\prime \prime}$ is higher than 1 indicating a very dominant elastic behaviour.

To provide further understanding of the phase transition behaviour of the GO dispersion, we measured the viscosity and calculated the ratio of elastic and loss moduli of the system with increasing the GO concentration (Fig. 4). As expected, both viscosity and $G^{\prime} / G^{\prime \prime}$ increase with GO concentration in the low concentration range until a peak is reached at $0.5 \mathrm{mg} \mathrm{ml}^{-1}$, then a sudden drop in both viscosity and $G^{\prime} / G^{\prime \prime}$ is observed. As we showed before, ${ }^{5}$ entropy plays an important role in 
the ordering of GO sheets. Upon becoming concentrated, the isotropic fluid of two dimensional disk-like GO sheets must undergo a transition to a nematic phase in which GO sheets encompass a preferred orientation. This orientational ordering consequently results in loss of orientational entropy. However, this loss of entropy is compensated with an increase in free volume. Therefore, there will be a net gain of entropy, as a result of the increase in free volume (packing) entropy. This sudden free volume expansion accompanied by ordering in the direction of shear, because of the spontaneous formation of LC domains, results in a sudden drop of viscosity and the ratio of elastic to viscous moduli. By increasing GO concentration furthermore, both viscosity and the moduli ratio gradually increase again until around $2.5 \mathrm{mg} \mathrm{ml}^{-1}$. However, at the concentration range of $2.5 \mathrm{mg} \mathrm{ml}^{-1}$ to $4 \mathrm{mg} \mathrm{ml}^{-1}$, a sudden drop of $G^{\prime} / G^{\prime \prime}$ can be observed suggesting a disturbance in the system. The latter might be due to an underlying phase transition with a tendency of the flakes to stack more regularly. Indeed, it has been observed that clays platelets can form columnar and hexagonal phases at high concentration. ${ }^{25}$ The formation of such phases can be prevented in the present case by the polydispersity of the system or by dynamical arrest; but the tendency of the flakes to pack more regularly could explain changes in rheological properties. At the critical concentration ( $4 \mathrm{mg} \mathrm{ml}^{-1}$ ), the increase in viscosity levels out and $G^{\prime} / G^{\prime \prime}$ ratio starts to increase again. Stacking of the GO mesogens results in further free volume expansion ${ }^{30}$ and subsequently hinders any increase in the viscosity (Fig. 1h).

\section{Conclusions}

The viscoelastic behaviour of this fascinating material offers significant features that can prove to be useful for both fundamental researches in two-dimensional materials and practical applications as demonstrated by a wide range of processing techniques employed in the present paper. We emphasize that the characteristic flow behaviour of LC GO is fundamentally different from those associated with usual viscoelastic materials including polymers. The generic properties reported here can be considered as a universal guideline to process different $\mathrm{GO}$ dispersions based on their rheological properties. Therefore, a simple rheological test and the comparison with the guideline provided here can assist others in the field to decide on what processing techniques should be employed and why.

\section{Notes and references}

${ }^{a}$ Intelligent Polymer Research Institute and ARC Centre of Excellence for Electromaterials Science and ${ }^{b}$ Institute for Superconducting and Electronic Materials, AIIM Facility, Innovation Campus, University of Wollongong, North Wollongong, NSW 2522, Australia

${ }^{c}$ Centre de Recherche Paul Pascal, Université Bordeaux I, UPR CNRS 8641, France

Email: gwallace@uow.edu.au

† Electronic Supplementary Information (ESI) available: [experimental, supporting discussions, supporting figures S1 to S5 and Table S1]. See DOI: $10.1039 / x 000000 \mathrm{x} /$

$\ddagger$ These authors contributed equally to this work.
The authors thank the Australian National Fabrication Facility (ANFF) Materials Node for their provision of research facilities. This work was supported by ARC Discovery Project DP1093952 (KK and SHA) and ARC Federation Fellowship (GGW) and ARC Professorial Fellowship (GMS).

1. R. Jalili, S. H. Aboutalebi, D. Esrafilzadeh, Shepherd Roderick L., Chen Jun, Aminorroaya-Yamini Sima, K. Konstantinov, Minett Andrew I. , J. M. Razal and G. G. Wallace, Adv. Funct. Mater., 2013, 23, 5344-5344.

2. S. H. Aboutalebi, M. M. Gudarzi, Q. B. Zheng and J.-K. Kim, Adv. Funct. Mater., 2011, 21, 2978-2988.

3. Z. Xu and C. Gao, ACS Nano, 2011, 5, 2908-2915.

4. J. E. Kim, T. H. Han, S. H. Lee, J. Y. Kim, C. W. Ahn, J. M. Yun and S. O. Kim, Angew. Chem. Int. Ed., 2011, 50, 3043-3047.

5. R. Jalili, S. H. Aboutalebi, D. Esrafilzadeh, K. Konstantinov, J. M. .Razal, S. E. Moulton and G. G. Wallace, Mater. Horiz., 2014, 1, 8791.

6. R. Jalili, S. H. Aboutalebi, D. Esrafilzadeh, K. Konstantinov, S. E. Moulton, J. M. Razal and G. G. Wallace, ACS Nano, 2013, 7, 39813990.

7. M. M. Gudarzi, M. H. M. Moghadam and F. Sharif, Carbon, 2013, 64, 403-415.

8. Z. Xu and C. Gao, Nat Commun., 2011, 2, 571.

9. C. Cheng and D. Li, Adv. Mater., 2013, 25, 13-30.

10. D. T. N. Chen, Q. Wen, P. A. Janmey, J. C. Crocker and A. G. Yodh, Annu. Rev. Cond. Mat. Phys., 2010, 1, 301-322.

11. J. R. Seth, L. Mohan, C. Locatelli-Champagne, M. Cloitre and R. T. Bonnecaze, Nat Mater., 2011, 10, 838-843.

12. G. Ovarlez, Q. Barral and P. Coussot, Nat Mater., 2010, 9, 115-119.

13. C. Storm, J. J. Pastore, F. C. MacKintosh, T. C. Lubensky and P. A. Janmey, Nature, 2005, 435, 191-194.

14. X. Li, G. Zhang, X. Bai, X. Sun, X. Wang, E. Wang and H. Dai, Nat. Nanotechnol., 2008, 3, 538-542.

15. Q. Zheng, W. H. Ip, X. Lin, N. Yousefi, K. K. Yeung, Z. Li and J.-K. Kim, ACS Nano, 2011, 5, 6039-6051.

16. A. Jaworek and A. T. Sobczyk, J. Electrostatics, 2008, 66, 197-219.

17. R. Mezzenga, C. Meyer, C. Servais, A. I. Romoscanu, L. Sagalowicz and R. C. Hayward, Langmuir, 2005, 21, 3322-3333.

18. B. Derby, Annu. Rev. Mater. Res., 2010, 40, 395-414.

19. F. Torrisi, T. Hasan, W. Wu, Z. Sun, A. Lombardo, T. S. Kulmala, G.-W. Hsieh, S. Jung, F. Bonaccorso, P. J. Paul, D. Chu and A. C. Ferrari, ACS Nano, 2012, 6, 2992-3006.

20. M. Kroon, W. L. Vos and G. H. Wegdam, Phys. Rev. E, 1998, 57, 1962-1970.

21. R. Jalili, J. M. Razal and G. G. Wallace, J. Mater. Chem., 2012, 22, 25174-25182.

22. R. Jalili, J. M. Razal, P. C. Innis and G. G. Wallace, Adv. Funct. Mater., 2011, 21, 3363-3370.

23. D. Esrafilzadeh, J. M. Razal, S. E. Moulton, E. M. Stewart and G. G. Wallace, J. Control. Release, 2013, 169, 313-320.

24. R. Jalili, J. M. Razal and G. G. Wallace, Sci. Rep., 2013, 3. 
25. M. C. D. Mourad, D. V. Byelov, A. V. Petukhov, D. A. Matthijs de Winter, A. J. Verkleij and H. N. W. Lekkerkerker, J. Phys. Chem. B, 2009, 113, 11604-11613.

26. P. Sollich, F. Lequeux, P. Hébraud and M. E. Cates, Phys. Rev. Lett., 1997, 78, 2020-2023.

27. L. J. Michot, C. Baravian, I. Bihannic, S. Maddi, C. Moyne, J. r. m. F. L. Duval, P. Levitz and P. Davidson, Langmuir, 2008, 25, 127139.

28. M. Zapotocky, L. Ramos, P. Poulin, T. C. Lubensky and D. A. Weitz, Science, 1999, 283, 209-212.

29. L. A. Hough, M. F. Islam, P. A. Janmey and A. G. Yodh, Phys. Rev. Lett., 2004, 93, 168102-168106.

30. H. H. Wensink and H. N. W. Lekkerkerker, Mol. Phys., 2009, 107, 2111-2118. 